\title{
Vorwort
}

\section{Eine Annäherung an das Solatium Podagricorum}

\begin{abstract}
„Seine spielende Gewandtheit im lateinischen Ausdruck, sein nie versiegender, durch reiche Erfahrung verschärfter Humor und das unablässige Drängen so mancher guter Freunde verleitete ihn gar oft, die Thorheiten seines Zeitalters launig durchzuhecheln, oder einen anscheinend widersinnigen Satz mit komischer Kraftanstrengung im Tempel der Weisheit aufzuhängen“ (Westermayer 1868, 216).
\end{abstract}

„ein [...] Meister des arguten Sprechens, dem nahezu jedes Wort zu einer Anspielung gerät“ (Breuer 1982, 60).

Mehr als für andere Werke Jakob Baldes (1604-1668) gilt für das Solatium podagricorum der Satz per aspera ad astra. Was Balde mit ,komischer Kraftanstrengung' geschaffen hat, erfordert beim Rezipienten ernsthaftes Bemühen. Dieser ist laufend aufgerufen, sich in einem dunklen Gelände zu orientieren und den Weg mit dem eigenen spärlichen Licht aufzufinden. Die Erfahrung, daß er dabei an manchen Stellen nicht weiterkommt, weil sein Licht verlischt, oder die Gefahr, daß er an anderen Stellen irrige Richtungen einschlägt, weil sein Licht sich als Irrlicht erweist, darf ihn nicht entmutigen. Er hat es hinzunehmen, daß nicht eines der 43 Prosastücke des ersten und nicht eines der 89 Gedichte des zweiten Buches bisher aufgehellt worden ist - auch nicht ansatzweise. Wie manche von Baldes Spätschriften wurde auch das Solatium podagricorum zur Gänze vergessen.

Im 16. und 17. Jahrhundert gab es eine reiche Podagraliteratur, nicht nur, wie es natürlich ist, medizinischer, sondern auch, wie es überraschend ist, literarischer Art. Zwei Bahnbrecher sind zu nennen, in indirekter Hinsicht Erasmus von Rotterdam, der mit dem ,Lob der Torheit' von 1509 ein Paradoxenkomium von breitester Wirkung schuf, das bei den Humanisten eine Vorliebe für die Behandlung paradoxer Thematiken begründete, und in direkter Hinsicht Willibald Pirckheimer, der mit der ,Verteidigung der Gicht ' von 1522 ein Podagraenkomium von gattungsbegründender Wirkung ersann, das dazu führte, daß in den folgenden 140 Jahren Autoren immer wieder das Podagra eigenwillig bewerteten und vor allem: ihm positive Seiten abzuwinnen versuchten. Heraus ragen in dieser Entwicklung Johann Carnarius (1553), Girolamo Cardano (1562), Johann Fischart (1577), Georg Fleissner (1596), Georg Barthold Pontanus (1605) - um nur diese zu 
nennen. Balde steht mit dem Solatium podagricorum von 1661 nicht nur am Ende der Gattung, sondern führt sie auch zu ihrem Höhepunkt.

Wirkt auf den modernen Rezipienten die Thematik abgelegen, ja verschroben, ist zu berücksichtigen, daß sie dem zeitgenössischen Leser brandaktuell erscheinen mußte. Was soll man sich unter dem Titel der Schrift vorstellen? Einen medizinischen Traktat? Eine medizinhistorisch relevante Erörterung? Einen christlichen Zuspruch? Es ist mit Nachdruck vorauszuschicken, daß es sich - auch im ersten in Prosa gehaltenen Teil - um kein medizinisches Fachbuch und demzufolge bei der vorgelegten Erklärung um keine medizinhistorische Arbeit handelt. Wer sich über den Stand der Podagraforschung im 17. Jahrhundert informieren möchte, greife besser zu den damals schon verbreiteten Sachbüchern. Das Solatium ist ,Literatur', die weitgehend Deutung vermitteln und die Weltanschauung ihres Dichters transportieren will. Man kann auch sagen: Nicht nur der zweite in Hexametern gehaltene Teil ist Dichtung, sondern über weite Strecken auch der erste. Deshalb sei der freundliche Leser - Balde würde mit den alten Römern sagen: der candidus lector, wir fügen hinzu: die candida lectrix - vor falschen Erwartungen gewarnt. Sicher wäre Balde erfreut gewesen, wenn er hätte annehmen dürfen, daß spätere Medizinhistoriker einzelne Fakten seiner Darstellung zu schätzen wüßten. Aber er hätte zuversichtlich gehofft, daß sie wahrnähmen, in welchem Maß diese Fakten in ein satirisches Umfeld eingebettet sind. Denn das Solatium ist eine Satire. Und eine Satire wurzelt nach römischer - vorbildlicher - Tradition, zumal aufgrund ihrer Abwehrhaltung, in einem durch und durch individuellen Grund: Nicht nur werden die Positionen anderer aus subjektiver Sicht dargestellt und bewertet, sondern auch eigene Probleme zur Darstellung gebracht. Allein die Frage der 13. Quaestio, ob die Schmerzen, die den Autor 1641 heimgesucht hatten, vom Podagra verursacht waren, zeigt, daß die persönliche Grundierung nicht zu unterschätzen ist.

Zwar wurden dem zweiten Buch selektive - vom philologischen Standpunkt aus allerdings unzureichende - Nachdichtungen von Liebhabern zuteil, aber die BaldeForschung ist an der Schrift achtlos vorbeigegangen. Westermayer (1868) widmete ihr knapp zwei Seiten, Bach (1904) kaum eine Seite, Stroh (2006) wenige Zeilen, und auch die von diesem und Katharina Kagerer im Internet betreute wertvolle Bibliographie nennt keine spezielle Abhandlung. Da ist es ein bemerkenswertes Ereignis, daß die Augsburger Postzeitung vom 29. April 1869 einen Auszug aus Baldes am Anfang der Schrift stehenden Brief an Thomas Barnazolius über seinen Besuch in Augsburg brachte ( $\bullet$ S. 107 Anm. 4) - doch auch dieser ist vergessen. „Außer einigen kulturhistorisch wichtigen Stellen hat das Gedicht für uns keinen Reiz mehr“, urteilte Bach (1904). Und doch tut sich ein zartes Licht in der Gegenwart auf: Kivistö (2009) zitiert in ihrer Darstellung medizinischer Thematik in der Neulateinischen Satire wenigstens einige Stellen aus dem Solatium in englischer Übersetzung. 
Gewiß, die Gicht ist heute nicht mehr eine allgemeine Plage. Aber wer erfahren wollte, wie sein Leiden erklärt und behandelt werden könne, las auch im 17. Jahrhundert nicht in erster Linie das Erzeugnis eines Dichters - zumal eines Satirikers, dessen Schriften extrem schwierig waren und vom Leser geistiges Bemühen und kombinierende Energie verlangten, um verstanden $\mathrm{zu}$ werden. Schon wegen der Sprache und der vorausgesetzten literarischen Bildung war nur eine Elite angesprochen. Doch wer wissen wollte, wie ein kluger Kopf die verbreitete Plage in kulturkritischer Hinsicht analysiert und bewertet, mußte sich angezogen fühlen von dem geistvollen Duktus der Argumentation, die Wege zu zeigen versuchte, das Leiden vor allem innerlich, mental zu bestehen - und eben das ist der Grund, weshalb das Solatium noch heute eine Beschäftigung mit ihm lohnt. Nur wer es nicht scheut, auf Schritt und Tritt Verborgenes zu entdecken, Verfremdetes zu klären, Verschlüsseltes zu entschlüsseln, wird die anspruchsvolle Schrift würdigen können und sich am Ende reich belohnt sehen.

Die Eigenart des Solatium bedingt die Anlage und den Charakter der vorliegenden Ausgabe. Diese verfolgt kein weiteres Ziel, als eine elementare Einführung in das in vielen Punkten dunkle Werk zu geben, als eine zum Originaltext hinführende wörtliche Übersetzung und einen einfachen Lesekommentar zu bieten, der dem Rezipienten eine erste Hilfestellung gibt. Sie hofft aber, daß dieser in die Lage versetzt wird, an dem Spinnen des Ariadnefadens, der ihn aus den Gängen des Labyrinths zum Licht der Erkenntnis führen soll, selbst mitzuwirken. Es werden nirgends ultima verba gesprochen, sondern durchweg vorsichtige Schritte unternommen. Die nachfolgenden Ausführungen können nicht mehr sein als eine Annäherung an die dunkle Schrift.

Bei einer Aufgabe könnten Kommentator und Rezipient des Solatium resignieren: bei dem Bemühen, die zahlreichen vorgeführten Personen zu identifizieren. Niemand kann annehmen, daß die Schrift im luftleeren Raum schwebt. Sie kontert scharf den Angriffen eines Sallius Gintrio und nimmt auch sonst auf Personen ihrer Gegenwart Bezug. Aber es ist wie bei Horaz, dessen Personal in den Satiren ebenfalls zwischen realer und fiktiver Welt angesiedelt ist. Was wir dem Augusteer des ersten vorchristlichen Jahrhunderts nachsehen, sollten wir auch dem Jesuiten des 17. Jahrhunderts nachsehen.

Originaltext: Die charakteristische Form, die dem Dichter vertraut war, wird beibehalten. Interpunktionen, Akzente oder Ligaturen sind ebenso übernommen wie der Laut- und Buchstabenbestand. Es ist ein Irrtum zu glauben, daß Baldes Werke in moderner Schreibweise leichter zu verstehen seien. In ihr sehen sie nur glatter aus, als sie sind - was ihrem Verständnis nicht guttut.

Übersetzung: Die deutsche Version soll das Original verstehen helfen, sie scheut sich nicht, das Deutsche dem Lateinischen anzunähern, sie verzichtet jedoch darauf, Verrenkungen zu produzieren, etwa den vom Deutschen abweichenden Gebrauch der Tempora (besonders 
des Plusquamperfekts und des Futur II) sowie der Komparative und Superlative in jedem Fall wiederzugeben. Es wird aber angestrebt, nicht falsche Glätte vorzutäuschen, sondern Wörtlichkeit und Lesbarkeit zu vereinen.

Interpretation (Kommentar): Die Erklärung verbindet sowohl bei den Quaestiones des ersten als auch bei den Gedichten des zweiten Buches eine Einbeziehung des Ganzen, die die jeweilige Aussage einzuordnen und die Zusammenhänge darzulegen versucht, mit der notwendigen Erhellung des Einzelnen, die öfter in die Anmerkungen verwiesen ist. Besonderer Wert wird auf die Herausstellung von Baldes Vorliebe gelegt, exquisite, auch abgelegene Stellen vor allem aus seinen bevorzugten Autoren wie Vergil, Horaz, Ovid (Metamorphosen), Lukan, Persius, Statius, Martial, Juvenal und Claudian aufzunehmen und nicht selten umzudeuten.

Abkürzungen: Ca. (= Castrum) und El. (= Elenchus) bezeichnen die beiden Teile der Expeditio polemico-poëtica (1664), auf deren Erklärung von 2017 immer wieder verwiesen wird, um viele Punkte nicht wiederholen zu müssen. Eindeutige Formulierungen helfen zwischen dem Elenchus der Expeditio und den Elenchi des Solatium zu unterscheiden (lediglich der erste wird hier kursiv gedruckt). Im Literaturverzeichnis genannte Werke werden mit den Nachnamen der Verfasser zitiert.

Verweise: Der dreieckige Pfeil • verweist nur auf Stellen in diesem Band.

,Podagra' (und Verwandtes): Es ist zu beachten, daß das Wort Podagra im Griechischen und Lateinischen weiblich, im Deutschen sächlich ist und daher zwischen dem lateinischen Text und der deutschen Übersetzung Spannungen bestehen. Wenn Balde an bestimmten Stellen auf das weibliche Geschlecht der Krankheit Wert legt, wird in der Übersetzung die Alternative ,Gicht verwendet. Ein vom Podagra Befallener heißt im Original podagricus, im Deutschen Podagrist, Podagrischer oder Podagraist; in dieser Arbeit wird auch das ältere Wort Podagriker gebraucht. Balde wertet im letzten Fuß des Hexameters die zweite Silbe von podagra / podagram als Länge (griechische Betonung bzw. lateinische Positionslänge). In 2, 10, 2 erscheinen spielerisch beide Messungen in einem Vers. Auch im fünften Fuß kann die zweite Silbe lang gemessen werden (so 2, 66, 1; 2, 76, 17). Martial mißt in seinem Podagragedicht 7, 39 (zu 2, 27 zitiert) am Versende ebenfalls podágram. Alle Formen von podagricus können im ersten und in der Synopsis zum zweiten Buch begegnen. In den Gedichten ist das bei drei bzw. vier aufeinanderfolgenden Kürzen nicht möglich (Ausnahmen 2, 21, 7 und 2, 49, 2 podágricus, 2, 44, 29 und 2, 87, 23 podágrica mit Positionslänge, jeweils im fünften Fuß). Öfter nimmt Balde eine eigenartige Metathese vor, indem er die ersten beiden Buchstaben umstellt und Opdagricus (2, 42, 21; 2, 47, 9; 2, 87, 22), Opdagricum $(2,62,7)$, Opdagrici $(2,24,2 ; 2,74,23)$ und Opdagricis $(2,4,2 ; 2,81,25)$ schreibt. Im ersten Buch und in der Synopsis des zweiten Buches begegnen diese Bildungen nicht. Es sind somit metrische Gründe maßgebend. Vielleicht hatte Balde dafür Anregungen bzw. Parallelen. Es mag sich aber auch um einen eigenen skurrilen Einfall handeln, da die fußgeschädigten Podagriker einen trippelnden / stolpernden Gang haben (auf den der veraltete Name ,Zipperlein` anspielt). Das könnte die Metathese versinnbildlichen. (Interessant ist, daß der Arzt Johann Heinrich Cohausen, einer der Nachschöpfer des zweiten Buches, in seiner Schnupftabaksatire $(\bullet$ S. 70) diese Form ohne metrischen Zwang aufnahm: ,Wie viel solte man wohl meinen, daß Sambricus Opdagricus mit seiner höckerichten Nase consumiret? [...] Wie viel Schnargutius, der eine stinckende hat $(1720,86)$. Auch den Namen Schnargutius könnte Cohausen Balde verdanken $(\bullet 2,88$, Nr. 8).

,Ballade': Dieser Begriff wird nicht in der Bedeutung des Terminus der Deutschen Literaturwissenschaft, sondern allgemein verwendet.

Frontispiz: Das Titelkupfer der Ausgabe von 1661 stammt von dem Augsburger Kupferstecher Melchior Küsell (1622-1683), der auch die Titelkupfer zur Satyra contra abusum tabaci (1657) und zum Magnus Tillius redivivus (Druck 1678) sowie sechs Kupfer zur Urania 
victrix (1663) schuf (Westermayer 1868, 254, 261-263). Es zeigt einen sitzenden Podagristen, der ein Kästchen mit Arzneimitteln ( $\bullet$ z. B. 1, 15 pilulas antipodagricas) in den Händen hält und zu dessen Füßen zwei Katzen kauern; er trägt stützende Stiefel (obwohl deren wärmespendende Funktion gefährlich sein kann: - 1, 16). An dem Baldachin des Bettes steht die bekannte Aufforderung Hic Rhodus, hic saltus, die allgemein etwa aussagt: ,Hier kommt es darauf an! / ,Beweise, was in dir steckt!' $(-2,55,12)$. (Soll der Angesprochene wieder springen? Oder nur tanzen? Denn die Podagriker können gute Tänzer sein: - 2, 24.) Zwei Arzneitöpfe tragen Aufschriften, der linke auf dem Tisch Patientia, die für den Gichtkranken so wichtig ist $(\bullet 1,13$ [1] profundissimum Patientiæ mysterium; Synopsis zu 2, 42 inuicta patientia; 2, 53 victrix patientia; 2, 60, 27 gaudet patientia duris [nach Lukan 9, 403]), der auf dem Baldachin Tamen est aliquid (was wohl bedeutet, daß es zur Heilung ,doch etwas gibt‘, also etwa auxilii oder pharmaci zu ergänzen). Dazu könnte das daneben hockende geflügelte Eichhörnchen passen, ein Tier, das verschiedene symbolische Bedeutungen hatte, hier vielleicht das der Beharrlichkeit / Ausdauer (Knacken einer harten Nuß o. ä.; „,mit seiner einmaligen Sprungkraft Wunsch- und Vorbild für den Podagristen: Hic, Rhodus... [Wilfried Stroh]) und Hoffnung (Flügel). Links vor dem Kranken steht ein leierspielender Jüngling, zu dessen Füßen ein Hahn (der auf Asklepios weist: > S. 243 Anm. 146) gebildet ist. Man könnte an Apollo denken, der Gott sowohl der Heilkunst als auch der Dichtkunst war $(\bullet$ 1, 39: Apollo Medicorum \& Poëtarum Præses). Aber der Heiligenschein legt eher den Bezug auf David nahe (Wilfried Stroh) und zwar auf 1 Reg (= 1 Sam der Lutherbibel) 16, 23 Igitur quandocumque spiritus Domini malus arripiebat Saul, David tollebat citharam, et percutiebat manu sua, et refocillabatur Saul, et levius habebat; recedebat enim ab eo spiritus malus (,Wenn nun der Geist Gottes über Saul kam, so nahm David die Harfe und spielte mit seiner Hand; so erquickte sich Saul, und es ward besser mit ihm, und der böse Geist wich von ihm', Luther). Die Stelle erklärt zugleich den spiritus ater der Bildunterschrift (gewissermaßen des Mottos des Buches), die wohl von Balde selbst stammt (Wilfried Stroh), bestehend aus zwei Hexametern: Expellam: si quis vexat te spiritus ater.| Humores, Curæq́ue solent mitescere cantu (,Ich werde es verbannen, wenn dich finsteres / trauriges Seufzen quält. | Säfte [des Podagra: - S. 11 Anm. 44] und Sorgen pflegen durch Gesang milder zu werden': - S. 23). spiritus hier wohl abgeschwächt ,Seufzen“ wie Hor. Epod. 11, 10 (Kießling / Heinze 1930, 531), mitescre cantu wie Calp. Sic. 3, 41 (Stefan Faller).

In vielen Fällen hat es kundige Wegweiser gegeben, denen an dieser Stelle aufrichtig gedankt sei. Hier werden nur die Namen genannt, vor Ort kommen sie mit ihren mündlich oder elektronisch vermittelten Auskünften zu Wort. Sie werden dort ohne Quellenangabe, aber mit ausgeschriebenen Vornamen zitiert, während sonst nur die Initialen angegeben sind. Dank gebührt Prof. Jochen Althoff (Mainz); Prof. Ulrike Auhagen (Freiburg); Prof. Andrea Bagordo (Freiburg); Prof. Anton Bierl (Basel); Prof. Jürgen Blänsdorf (Mainz); Prof. Jan Bloemendal (Amsterdam); Prof. Karl Heinz Burmeister (Bregenz / Lindau); Prof. Carmen Cardelle de Hartmann (Zürich); Prof. Klaus Döring (Bamberg / Freiburg); Prof. Karl Enenkel (Münster); Dr. Stefan Faller (Freiburg); Prof. John L. Flood (London); Prof. Hans-Joachim Gehrke (Freiburg); Prof. Giovanna Gianola (Padova); Prof. Anja Grebe (Krems / Donau); Dr. Mirko Hanke (Deutsche Akademie der Naturforscher Leopoldina, Halle); Prof. Frank-Rutger Hausmann (Freiburg); Dr. Ingrid 
Helber (Balingen); Prof. Wolfgang Hübner (Münster); Prof. Klaus Jacobi (Freiburg); Prof. Roland Kany (München); Dr. Gabriele Kaps (Neuburg); Prof. Wolfgang Kofler (Innsbruck); Prof. Severin Koster (Erlangen); Prof. Hartmut Laufhütte (Passau); Prof. Marc Laureys (Bonn); Prof. Virginie Leroux (Paris); Prof. Detlef Liebs (Freiburg); Prof. Walther Ludwig (Hamburg); Dipl. Bibl. Wolfgang Mayer (Staats- und Stadtbibliothek Augsburg); Prof. Wolfgang Mieder (Burlington, Vermont); Prof. Gernot Michael Müller (Bonn); Prof. Gabrielle Oberhänsli (Freiburg); Prof. Eckart Olshausen (Stuttgart); Prof. Johannes Platschek (München); Prof. Peter Pörtner (München); Prof. Joachim Friedrich Quack (Heidelberg); Dr. Christian Quaeitzsch (Bayer. Verwaltung der staatlichen Schlösser, Gärten und Seen, Museumsabteilung, München); Staatsarchivar a. D. Dr. Reinhard H. Seitz (Neuburg); Dr. Andrea Sommer-Mathis (Österr. Akad. der Wiss., Institut für Kulturwiss. und Theatergesch., Wien); Prof. Volker Michael Strocka (Freiburg); Prof. Wilfried Stroh (München); Prof. Stefan Tilg (Freiburg); Prof. Kai Trampedach (Heidelberg); Dr. Tobias Uhle (München); Prof. Peter Walter † (Freiburg); Prof. Claudia Wiener (München); Prof. Aloys Winterling (Berlin); Doris Wittmann (Stadtmuseum Ingolstadt); Dr. Gabriele Wolf (Bayer. Akad. der Wiss., Institut für Volkskunde, München); Dr. Heike Zech (Germ. Nationalmuseum Nürnberg).

Die Abhandlung ist dem Andenken der am 7. Mai 2018 gestorbenen Frau des Verfassers, der Ärztin Helga Lefèvre, gewidmet, die mehrere Jahre Assistentin am Medizinhistorischen Institut der Universität Kiel war und ihn in über 50 Jahren in medizinische und medizinhistorische Probleme eingeführt hat und auch jetzt wieder bei den Grundlagen eine geduldige Wegweiserin gewesen ist.

Geschrieben in den Jahren 2017 / 2019 in Freiburg-Ebnet mit Blick aus dem Arbeitszimmer auf den nahen Schwarzwald, auf die aufgehende Sonne und den aufgehenden Mond, auf die direkt vor dem Fenster spazierenden Störche und Reiher und ausgestattet mit der Reproduktion eines Exemplars des Solatium podagricorum aus der ehemaligen Benediktinerabtei St. Peter, die nach wenigen Kilometern zu erreichen ist.

Großer Dank gilt wiederum den Herausgebern der ,Beiträge‘, vor allem Prof. Dr. Michael Erler und Prof. Dr. Clemens Zintzen, für die Aufnahme der Abhandlung in die angesehene Reihe, ferner dem Verlag Walter de Gruyter, der den Band kompetent und großzügig erstellt hat, besonders Dr. Mirko Vonderstein für freundliches Entgegenkommen und Florian Ruppenstein für den gewohnt vorzüglichen Satz, Dr. Christian Hänger (Univ.-Bibl. Mannheim) für die eigens angefertigte CD mit dem Frontispiz der Ausgabe von 1661 sowie Dr. Stefan Faller (Freiburg), der nicht nur erneut Hilfe im Umgang mit dem Computer geleistet, sondern auch zahlreiche fachliche Auskünfte gegeben und die Appendix 2 verfaßt hat. 